\title{
Vector-exponential time-series modeling for polynomial $J$-spectral factorization
}

\author{
I. Pendharkar, H. Pillai
}

\author{
P. Rapisarda
}

\begin{abstract}
An iterative algorithm to perform the $J$-spectral factorization of a para-Hermitian matrix is presented. The algorithm proceeds by computing a special kernel representation of an interpolant for a sequence of points and associated directions determined from the spectral zeroes of the to-befactored matrix.
\end{abstract}

\section{INTRODUCTION}

The problem of polynomial $J$-spectral factorization is the following: a real para-hermitian $\mathrm{w} \times \mathrm{w}$ polynomial matrix $Z$ in the indeterminate $\xi$ (i.e. $Z(-\xi)=Z(-\xi)^{T}$ ) is given, together with two integers $n_{+}$and $n_{-}$such that $n_{+}+n_{-}=$w. It is required to find, if it exists, a $\mathrm{w} \times \mathrm{w}$ polynomial matrix $F$ such that

$$
Z(\xi)=F^{T}(-\xi) J F(\xi)
$$

where

$$
J=\left[\begin{array}{cc}
I_{n_{+}} & 0 \\
0 & -I_{n_{-}}
\end{array}\right]
$$

and $F$ is Hurwitz, i.e. has no singularities in the closed complex right half-plane.

Polynomial $J$-spectral factorization arises in different areas of systems and control, for example in the case of Wiener filtering, LQG theory, in the polynomial- and in the behavioral approach to $H_{\infty}$-control and filtering (see [13], [14], [15]. Many algorithms have been suggested for the solution of such problem, especially in the case when $n_{-}=0$, i.e. $J=I_{\mathrm{w}}$ (see [2], [4], [5], [6], [7], [8], [14], [21]). In this paper we propose an algorithm based on the $J$-unitary kernel representations of solutions to the subspace Nevanlinna interpolation problem (see [20]), and on the calculus of quadratic differential forms introduced in [23]. The theory of metric interpolation has been used in [6], [7] for solving the problem of rational spectral factorization (i.e. the case in which $J=I_{\mathrm{w}}$ and the entries of the matrix $Z$ consist of rational functions); however, the approach proposed in this paper differs from that of [6], [7] in many aspects. First, our approach arises in the theory of quadratic differential forms (QDFs in the following), and uses twovariable polynomial matrix algebra. This point of view allows new insights in the nature of the problem. For example, an important consequence of our reliance on the theory of QDFs is that we are able to formulate necessary and sufficient conditions for the existence of a $J$-spectral factorization based on the signature of a Pick-type matrix derived from $Z$ and its singularities in $\mathbb{C}_{+}$, thus providing an original and effective test alternative to the ones already known. Secondly, our approach covers also the case when $J \neq I_{\mathrm{w}}$, which is of special interest in $H_{\infty}$-control and filtering. Finally, the functioning of the algorithm does not depend on the assumptions underlying the algorithm proposed in [6], [7].

The paper is organized as follows: in section II we illustrate the basic features of modeling vector-exponential time series. In section III we illustrate our procedure for $J$-spectral factorization and we sketch the proof of its correctness. In section IV we illustrate the results with two examples. Section V is devoted to relating the results presented in section III to existing approaches, and to discussing the current extension of the results in several important directions.

Because of space limitations, we are forced to omit any exposition of the behavioral approach to systems and control, and of the theory of quadratic differential forms and the associated calculus of two-variable polynomial matrices; the interested reader is referred to [16], [23] respectively for a thorough exposition.

Notation: The space of $\mathrm{n}$ dimensional real, respectively complex, vectors is denoted by $\mathbb{R}^{\mathrm{n}}$, respectively $\mathbb{C}^{\mathrm{n}}$, and the space of $m \times n$ real, respectively complex, matrices, by $\mathbb{R}^{m \times n}$, respectively $\mathbb{C}^{\mathrm{m} \times \mathrm{n}}$. Whenever one of the two dimensions is not specified, a bullet $\bullet$ is used. Given two column vectors $x$ and $y$, we denote with $\operatorname{col}(x, y)$ the vector obtained by stacking $x$ over $y$; a similar convention holds for the stacking of matrices with the same number of columns. Given a Hermitian matrix $S \in \mathbb{C}^{\text {w } \times \mathrm{w}}$, we define its inertia as the triple $\sigma(S):=\left(\sigma_{-}, \sigma_{0}, \sigma_{+}\right)$where $\sigma_{+}$is the number of eigenvalues of $S$ with positive real part, $\sigma_{-}$is the number of eigenvalues of $S$ with negative real part, and $\sigma_{0}$ is the number of purely imaginary eigenvalues of $S$. If $A=\left[A_{i j}\right] \in \mathbb{C}^{\mathrm{p} \times \mathrm{m}}$, then $A^{*}:=\left[\overline{A_{j i}}\right] \in \mathbb{C}^{\mathrm{m} \times \mathrm{p}}$ with $^{-}$denoting complex conjugate.

The ring of polynomials with real coefficients in the indeterminate $\xi$ is denoted by $\mathbb{R}[\xi]$; the ring of two-variable polynomials with real coefficients in the indeterminates $\zeta$ and $\eta$ is denoted by $\mathbb{R}[\zeta, \eta]$. The space of all $\mathrm{n} \times \mathrm{m}$ polynomial matrices in the indeterminate $\xi$ is denoted by $\mathbb{R}^{\mathrm{n} \times \mathrm{m}}[\xi]$, and that consisting of all $\mathrm{n} \times \mathrm{m}$ polynomial matrices in the indeterminates $\zeta$ and $\eta$ by $\mathbb{R}^{\mathrm{n} \times \mathrm{m}}[\zeta, \eta]$. Given a matrix $R \in$ $\mathbb{R}^{\mathrm{n} \times \mathrm{m}}[\xi]$, we define $R(\xi)^{\sim}:=R(-\xi)^{T} \in \mathbb{R}^{\mathrm{m} \times \mathrm{n}}[\xi]$.

We denote with $\mathfrak{C}^{\infty}\left(\mathbb{R}, \mathbb{R}^{w}\right)$ the set of infinitely often differentiable functions from $\mathbb{R}$ to $\mathbb{R}^{\mathrm{w}}$. The exponential function $e^{\lambda t}$ is denoted with $\exp _{\lambda}$.

\section{II. $\Sigma$-UNITARY MODELING OF DUALIZED DATA SETS}

In this section we illustrate the basic features of modeling dualized data sets, a concept introduced in [20] in the context of the subspace Nevanlinna interpolation problem (SNIP in the following). We put special emphasis on the structure 
of the $J$-unitary kernel representation of its solution, which plays a central role in the algorithm for $\Sigma$-spectral factorization illustrated in section III. The interested reader is referred to [20] for a thorough exposition of the various aspects of the SNIP and to [3], [22] for information on the notion of Most Poweful Unfalsified Model and on how interpolation problems can be cast in the framework of modeling vectorexponential time-series.

A standard problem in modeling vector-exponential time series is the following (see [3], [20]): we are given $N$ distinct points $\lambda_{i}$ in the complex right half-plane, together with $N$ subspaces $\mathcal{V}_{i} \subseteq \mathbb{R}^{\mathrm{p}+\mathrm{m}}, i=1, \ldots, N$. It is required to find a polynomial matrix $R \in \mathbb{R}^{\mathrm{p} \times(\mathrm{p}+\mathrm{m})}[\xi]$, such that $R\left(\lambda_{i}\right) v=0$ for all $v \in \mathcal{V}_{i}, i=1, \ldots, N$; such a matrix $R$ is called a solution to the interpolation problem. Often additional requirements on $R$ are given, for example that the transfer function of the behavior $\operatorname{ker}\left(R\left(\frac{d}{d t}\right)\right)$ is stable and contractive, as it happens in the SNIP.

In order to state the main result of this section we need first to introduce a couple of important concepts. The first one is that of data dualization. Define the matrix

$$
\Sigma:=\left[\begin{array}{cc}
I_{\mathrm{m}} & 0 \\
0 & -I_{\mathrm{p}}
\end{array}\right]
$$

We associate with each pair $\left(\lambda_{i}, \mathcal{V}_{i}\right)$ the set of trajectories

$$
\mathcal{V}_{i} \exp _{\lambda_{i}}:=\left\{v \exp _{\lambda_{i}} \mid v \in \mathcal{V}_{i}\right\}
$$

and we define its dual set as

$$
\mathcal{V}_{i}^{\perp} \exp _{-\bar{\lambda}_{i}}:=\left\{w \exp _{-\overline{\lambda_{i}}} \mid w \Sigma v=0 \text { for all } v \in \mathcal{V}_{i}\right\}
$$

We call the set

$$
\cup_{i=1}^{N} \mathcal{V}_{i} \exp _{\lambda_{i}} \cup \mathcal{V}_{i}^{\perp} \exp _{-\bar{\lambda}_{i}}
$$

the dualized data set.

Next, we introduce the notion of Pick matrix associated with the data $\left\{\left(\lambda_{i}, \mathcal{V}_{i}\right)\right\}_{i=1, \ldots, N}$. Let $V_{i} \in \mathbb{R}^{(\mathrm{p}+\mathrm{m}) \times \operatorname{dim}\left(\mathcal{V}_{i}\right)}$ be a full column rank matrix such that $\operatorname{Im}\left(V_{i}\right)=\mathcal{V}_{i}, i=$ $1, \ldots, N$. The $\left(\sum_{i=1}^{N} \operatorname{dim}\left(\mathcal{V}_{i}\right)\right) \times\left(\sum_{i=1}^{N} \operatorname{dim}\left(\mathcal{V}_{i}\right)\right)$ Hermitian matrix

$$
T_{\left\{\mathcal{V}_{i}\right\}}:=\left[\frac{V_{i}^{*} \Sigma V_{j}}{\lambda_{i}+\lambda_{j}}\right]_{i, j=1, \ldots, N}
$$

is called the Pick matrix associated with $\left\{\left(\lambda_{i}, \mathcal{V}_{i}\right)\right\}_{i=1, \ldots, N}$. Of course $T_{\left\{\mathcal{V}_{i}\right\}}$ depends on the particular basis matrices $v_{i}$ chosen for $\mathcal{V}_{i}$, but the signature of all such matrices is the same. It is indeed the signature that plays an important role in the algorithm for $J$-spectral factorization that will be stated in the next section.

The following result can be proved in a way analogous to that of Theorem 4.1 of [20].

Theorem 1: The following statements are equivalent:

1) The Hermitian matrix $T_{\left\{\mathcal{V}_{i}\right\}}$ is nonsingular;

2) The Most Powerful Unfalsified Model for the dualized data set $\cup_{i=1}^{N} \mathcal{V}_{i} \exp _{\lambda_{i}} \cup \mathcal{V}_{i}^{\perp} \exp _{-\overline{\lambda_{i}}}$ has a kernel representation of the form

$$
\hat{R}:=\left[\begin{array}{c}
M^{\sim} \Sigma \\
R
\end{array}\right]
$$

where $M \in \mathbb{R}^{(\mathrm{p}+\mathrm{m}) \times \mathrm{m}}[\xi], R \in \mathbb{R}^{\mathrm{p} \times(\mathrm{p}+\mathrm{m})}[\xi]$ satisfy the following properties:

(a) $R M=0$

(b) $\quad \hat{R}^{\sim} \Sigma \hat{R}=\hat{R} \Sigma \hat{R}^{\sim}=p p^{\sim} \Sigma$ with $p \in \mathbb{R}[\xi]$ a Hurwitz polynomial.

A matrix $\hat{R}$ satisfying the property $(b)$ in statement 2 of the Theorem will be called a $\Sigma$-unitary matrix in the following.

It can be shown (see the proof of Theorem 4.1 in [20] for details) that if the matrix $T_{\mathcal{V}}=\frac{V^{*} \Sigma V}{\bar{\lambda}+\lambda}$ is nonsingular, then given a subspace $\mathcal{V} \exp _{\lambda}$, a $\Sigma$-unitary model $\hat{R}$ satisfying properties $(a)-(b)$ of the Theorem can be computed as

$$
\hat{R}(\xi):=(\xi+\bar{\lambda}) I_{\mathrm{p}+\mathrm{m}}-V T_{\mathcal{V}}^{-1} V^{*} \Sigma
$$

where $V \in \mathbb{R}^{(\mathrm{p}+\mathrm{m}) \times \operatorname{dim}(\mathcal{V})}$ is such that $\operatorname{Im}(V)=\mathcal{V}$ and $T_{\mathcal{V}}$ is the Pick matrix associated with $\mathcal{V}$, i.e. $T_{\mathcal{V}}=\frac{V^{*} \Sigma V}{\lambda+\lambda}$. The partition (1) of $\hat{R}$ is obtained defining $M^{\sim} \Sigma$ and $R$ as the first $\mathrm{m}$ rows, respectively last $\mathrm{p}$ rows, of $\hat{R}$.

Using the representation (2) for the model for one set of trajectories $\mathcal{V} \exp _{\lambda}$ and the notion of error trajectory defined in [3], a recursive algorithm can be constructed that provides a representation for the model for $\cup_{i=1}^{N} \mathcal{V}_{i} \exp _{\lambda_{i}} \cup \mathcal{V}_{i}^{\perp} \exp _{-\overline{\lambda_{i}}}$ satisfying $(a)-(b)$ of Theorem 1 . See [20] for details.

\section{THE ALGORITHM}

In this section we illustrate an algorithm for the computation of a $J$-spectral factor of a para-Hermitian matrix $Z \in \mathbb{R}^{(\mathrm{p}+\mathrm{m}) \times(\mathrm{p}+\mathrm{m})}[\xi]$ based on the concept of $\Sigma$-unitary modeling illustrated in the previous section. In the following we present a simplified version of the algorithm for ease of exposition; for pedagogical reasons we leave out many of the technical details necessary in order to prove the general case. See also the remarks in section $\mathrm{V}$ of this paper.

We assume that a symmetric two-variable polynomial matrix $\Phi \in \mathbb{R}_{s}^{(\mathrm{p}+\mathrm{m}) \times(\mathrm{p}+\mathrm{m})}[\zeta, \eta]$ such that $\Phi(-\xi, \xi)=Z(\xi) \in$ $\mathbb{R}^{(\mathrm{p}+\mathrm{m}) \times(\mathrm{p}+\mathrm{m})}[\xi]$ has been computed, and that $\Phi(\zeta, \eta)$ admits the symmetric factorization (see [23] for details)

$$
\Phi(\zeta, \eta)=K^{T}(\zeta) \Sigma K(\eta)
$$

where $\Sigma \in \mathbb{R}^{\bullet \times \bullet}$ is a nonsingular symmetric matrix, and $K=\operatorname{col}(Q, P)$, with $Q \in \mathbb{R}^{\mathrm{m} \times(\mathrm{m})}[\xi], P \in \mathbb{R}^{\mathrm{p} \times(\mathrm{m})}[\xi]$, is such that $P Q^{-1}$ is a matrix of strictly proper rational functions. Observe that often, and most notably in the context of $\mathrm{H}_{\infty^{-}}$ control and filtering applications, the para-Hermitian matrix $Z$ actually is already given in such a factorized form (see for example [24], [25] and section 3 of [21], in particular Lemma 3.1 therein).

Now let $\lambda_{i}, i=1, \ldots, n$ be the singularities of $Z$ in the right half-plane, and let $V_{i} \in \mathbb{R}^{\bullet} \bullet$ be full column rank matrices such that $\operatorname{Im}\left(V_{i}\right)=\operatorname{Im}\left(K\left(\lambda_{i}\right)\right), i=1, \ldots, n$. In the following we make the assumptions:

1) $T=\left[\frac{V_{i}^{*} \Sigma V_{i}}{\lambda_{j}+\lambda_{i}}\right]_{i, j=1}^{n}$ is nonsingular;

2) $\operatorname{deg}(\operatorname{det}(Q))=n=\frac{1}{2} \operatorname{deg}(\operatorname{det}(Z))$.

Now partition $\Sigma$ as $\left[\begin{array}{cc}\Sigma_{1} & \Sigma_{2} \\ \Sigma_{2}^{T} & \Sigma_{3}\end{array}\right]$ compatibly with the partition of $K$; under the assumptions stated above, it can be 
shown that the inertia $\sigma(Z(i \omega))$ is constant for all $\omega \in \mathbb{R}$, and that it is the same as $\sigma\left(\Sigma_{1}\right)$. Observe that this implies that $Z$ admits a $\sigma\left(\Sigma_{1}\right)$-spectral factorization $Z=D^{\sim} \sigma\left(\Sigma_{1}\right) D$ (see [5]).

We now proceed first to state our algorithm for the computation of a $\Sigma_{1}$-spectral factorization of $Z$, i.e. of a square, Hurwitz matrix $D$ such that $Z=D^{\sim} \Sigma_{1} D$. Observe that once such factorization is known, the computation of a $\sigma\left(\Sigma_{1}\right)$-factorization of $Z$ can be computed easily from a factorization $\Sigma_{1}=V^{T} \sigma\left(\Sigma_{1}\right) V$ obtained through standard numerical procedures. After having stated the algorithm, we will sketch the line of proof to follow in order to show the correctness of the procedure.

\section{Algorithm 2:}

Input: $\Sigma, \quad K$ from a factorization $K^{\sim} \Sigma K$ of $Z$ satisfying Assumptions 1 and 2.

Output: A $\Sigma_{1}$-spectral factorization $Z=D^{\sim} \Sigma_{1} D$, with $D$ square and Hurwitz.

$$
\begin{aligned}
& \text { Compute the singularities } \lambda_{i}, i= \\
& 1, \ldots, n \text { of } Z \text { in } \mathbb{C}_{+} ; \\
& \text {Define } K_{1}:=K ; \\
& \text { For } i=1, \ldots, N \text { do } \\
& \quad \text { Let } V_{i} \in \mathbb{R}^{(\mathrm{p}+\mathrm{m}) \times \bullet} \text { be a full } \\
& \text { column rank matrix such that } \\
& \operatorname{Im}\left(V_{i}\right)=\operatorname{Im}\left(K_{i}\left(\lambda_{i}\right)\right) ; \\
& \quad \text { Compute model }(2) \quad R_{i} \\
& \mathbb{R}^{(\mathrm{p}+\mathrm{m}) \times(\mathrm{p}+\mathrm{m})}[\xi] \\
& \text { problem with data }\left(\lambda_{i}, V_{i}\right) ; \\
& \quad \text { Define } K_{i+1}(\xi):=\frac{R_{i}(\xi) K_{i}(\xi)}{\xi-\lambda_{i}} ; \\
& \text { end; }
\end{aligned}
$$

The following is the main result of this paper.

Theorem 3: Let $\Phi(\zeta, \eta)=K^{T}(\zeta) \Sigma K(\eta)$ be given, with $K=\operatorname{col}(Q, P) \in \mathbb{R}^{(\mathrm{p}+\mathrm{m}) \times \mathrm{m}}[\xi]$ such that $P Q^{-1}$ is a matrix of strictly proper rational functions. Denote the right halfplane zeros of $\operatorname{det}(\Phi(-\xi, \xi))$ with $\lambda_{i}, i=1, \ldots, n$, and let $V_{i} \in \mathbb{R}^{\bullet \times \bullet}$ be a full column rank matrix such that $\operatorname{Im}\left(V_{i}\right)=$ $\operatorname{Im}\left(K\left(\lambda_{i}\right)\right)$. Assume that conditions (1)-(2) are satisfied.

Then the matrix $K_{n+1}$ obtained at the end of Algorithm 2 is such that $K_{n+1}=\operatorname{col}(D, 0)$, with $D \in \mathbb{R}^{(\mathrm{m}) \times(\mathrm{m})}[\xi]$ a Hurwitz $\Sigma_{1}$-spectral factor of $\Phi(-\xi, \xi)$, i.e.

$$
\Phi(-\xi, \xi)=D(-\xi)^{T} \Sigma_{1} D(\xi)
$$

The proof of this result is rather lengthy and consequently in the following we merely sketch it, glossing over the many technical details.

Sketch of proof: First, it is shown that the nonsingularity of the Pick matrix $T=\left[\frac{V_{i}^{*} \Sigma V_{i}}{\lambda_{j}+\lambda_{i}}\right]_{i, j=1}^{n}$ is equivalent to the nonsingularity of the Pick matrix associated with the error subspace of the model (2) (see [3]) at the $i$-th iteration. This implies that the one-step model (2) can be defined at each iteration step.

The model (2) can also be shown to preserve the strict properness of $K_{i+1}$ in the step $K_{i}(\xi) \rightarrow \frac{R_{i}(\xi) K_{i}(\xi)}{\xi-\lambda_{i}}=$ $K_{i+1}(\xi)$ of the algorithm, in the following sense. If $K_{i}$ is such that $K_{i}=\operatorname{col}\left(Q_{i}, P_{i}\right)$ with $P_{i} Q_{i}^{-1}$ strictly proper, then also $K_{i+1}=: \operatorname{col}\left(Q_{i+1}, P_{i+1}\right)$ is such that $P_{i+1} Q_{i+1}^{-1}$ is strictly proper.

Now write $K_{i}=K_{i}^{\prime} U_{i}$, with $K_{i}^{\prime}$ right prime, and $U_{i} \in$ $\mathbb{C}^{\bullet} \times[\xi]$ a greatest common right divisor of $K_{i}$. Partition $K_{i}^{\prime}$ as $K_{i}^{\prime}=\operatorname{col}\left(Q_{i}^{\prime}, P_{i}^{\prime}\right)$. It can be shown that the degree of the determinant of the denominator $Q_{i}$ associated with the transfer function of $K_{i}$ decreases with $i$. In particular, it can be proved that $\operatorname{deg}\left(\operatorname{det}\left(Q_{n+1}^{\prime}\right)=0\right.$, i.e. $Q_{n+1}$ is a unimodular matrix. The last conclusion, together with the fact that the strict properness of of $K_{i}$ is preserved, implies that $P_{n+1}$ is the zero matrix.

It turns out that, due to the $\Sigma_{1}$-unitariness of the model (2), the matrix $K_{i}$ generated at each step satisfies

$$
K_{i}^{T}(-\xi) \Sigma K_{i}(\xi)=\Phi(-\xi, \xi)=Z(\xi)
$$

for $i=1 \ldots n$. Moreover, $\operatorname{deg}\left(\operatorname{det}\left(Q_{i}\right)\right)=\operatorname{deg}\left(\operatorname{det}\left(Q_{1}\right)\right)$, $i=1, \ldots, n$.

Now using the equation $K_{i}^{T}(-\xi) \Sigma K_{i}(\xi)=\Phi(-\xi, \xi)$ it can be shown that $Q_{n+1}^{\prime}$ satisfies $Q_{n+1}^{\prime}(-\xi)^{T} \Sigma_{1} Q_{n+1}^{\prime}(\xi)=$ $\Sigma_{1}$, i.e. $Q_{n+1}^{\prime}$ is unimodular and $\Sigma_{1}$-unitary. This implies that the product of the greatest common right divisors $U:=\prod_{i=1}^{n} U_{i}$ is Hurwitz, and consequently that the matrix $Q_{n+1}=Q_{n+1}^{\prime} U$ is a $\left(\Sigma_{1}\right)$-spectral factor of $\Phi(-\xi, \xi)=$ $Z(\xi)$ as stated in the claim of the Theorem.

\section{EXAMPLES}

In this section we provide two examples of the application of our results to $J$-spectral factorization. In the first one, Algorithm 2 can be applied as it is, since the conditions on the matrix $K$ arising from a symmetric canonical factorization of $\Phi$ are satisfied. In the second example, we show some of the preprocessing steps that need to be performed in order for the results to be applied to matrices not satisfying those conditions.

Example 4: Consider

$$
Z(\xi)=\left[\begin{array}{cc}
1-\xi^{2} & \xi \\
-\xi & 1-\xi^{2}
\end{array}\right]
$$

Note that $Z(i \omega)$ has inertia $(2,0,0)$ for all $\omega \in \mathbb{R}$. Pre-factor $Z(\xi)$ as

$$
Z(\xi)=\left[\begin{array}{cc}
-\xi & 0 \\
0 & -\xi \\
1 & 0 \\
0 & 1
\end{array}\right]^{T}\left[\begin{array}{cccc}
1 & 0 & 0 & -0.5 \\
0 & 1 & 0.5 & 0 \\
0 & 0.5 & 1 & 0 \\
-0.5 & 0 & 0 & 1
\end{array}\right]\left[\begin{array}{ll}
\xi & 0 \\
0 & \xi \\
1 & 0 \\
0 & 1
\end{array}\right]
$$

Observe that in this case $\mathrm{m}=\mathrm{p}=2$, that $\Sigma_{1}=I_{2}$, and that $n=\frac{1}{2} \operatorname{deg}(\operatorname{det}(Z))=2$. Observe also that $Z(\xi)$ has $0.86 \pm i 0.5$ as singularities in $\mathbb{C}^{+}$, and that since the inertia of $\Sigma_{1}$, the $(1,1)$-block of the matrix $\Sigma$, is $(2,0,0)$, there exists a $I_{2}$-spectral factorization of $Z$.

It can be easily verified that the Pick matrix associated with the data is nonsingular. Consequently the model (2) can be constructed at every step. We initialize

$$
K_{1}(\xi)=\left[\begin{array}{ll}
\xi & 0 \\
0 & \xi \\
1 & 0 \\
0 & 1
\end{array}\right]
$$


and we compute $K_{1}(0.86+i 0.5)$. It is not difficult to see that $K_{1}(0.86+i 0.5)$ has full column rank, and consequently we can define

$$
V_{1}=K_{1}(0.86+i 0.5)=\left[\begin{array}{cc}
0.86+i 0.5 & 0 \\
0 & 0.86+i 0.5 \\
1 & 0 \\
0 & 1
\end{array}\right]
$$

In step $i=1$, we proceed to compute a model $\hat{R}_{1}(\xi)$ as in (2) for the data pair $\left(0.86+i 0.5, \operatorname{Im}\left(V_{1}\right)\right)$ :

$$
\left[\begin{array}{cccc}
-i 0.6+\xi & -0.4 & -0.8-i 0.34 & 0.34+i 0.2 \\
0.4 & -i 0.6+\xi & -0.34-i 0.2 & -0.8-i 0.34 \\
-0.8+i 0.34 & -0.34+i 0.2 & -i 0.4+\xi & 0.4 \\
0.34-i 0.2 & -0.8+i 0.34 & -0.4 & -i 0.4+\xi
\end{array}\right]
$$

This model yields $K_{2}(\xi)=\hat{R}_{1}(\xi) K_{1}(\xi) /(\xi-0.86-i 0.5)$. It is easy to verify that $K_{2}(0.86-i 0.5)$ is also full column rank, and consequently we can define $V_{2}=K_{2}(0.86-i 0.5)$.

In step $i=2$, we compute a model $\hat{R}_{2}(\xi)$ as in (2) for the data pair $\left(0.86-i 0.5, \operatorname{Im}\left(V_{2}\right)\right)$ :

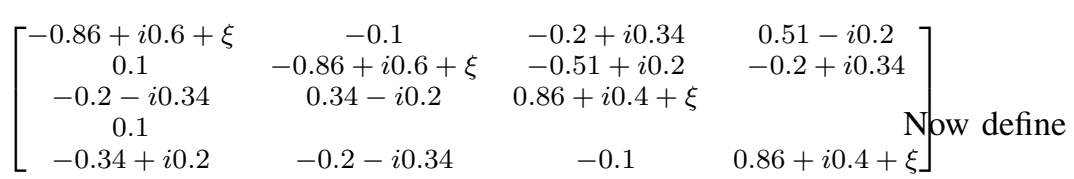

and

$$
M^{\prime}(\xi):=\left[\begin{array}{ccc}
1 & 0 & 0 \\
0 & 1 & 0 \\
-2 & 0 & 1
\end{array}\right] M(\xi)=\left[\begin{array}{cc}
1 & -1-\xi \\
-1 & 0 \\
0 & 2
\end{array}\right]
$$

Note that the transfer function corresponding to $M^{\prime}$ is strictly proper. Note also that $Z(\xi)=M^{\prime}(\xi)^{T} \Sigma^{\prime} M(\xi)$ where

$$
\Sigma^{\prime}=\left[\begin{array}{ccc}
1 & 0 & 0 \\
0 & 1 & 0 \\
-2 & 0 & 1
\end{array}\right]^{-T}\left[\begin{array}{ccc}
-3 & 0 & 2 \\
0 & 1 & 0 \\
2 & 0 & -1
\end{array}\right]\left[\begin{array}{ccc}
1 & 0 & 0 \\
0 & 1 & 0 \\
-2 & 0 & 1
\end{array}\right]^{-1}
$$

It is easy to verify that $M^{\prime}(1)$ has full column rank. We can choose $V_{1}=M^{\prime}(1)$ and proceed with the $\Sigma^{\prime}$ unitary modeling obtaining

$$
R(\xi)=\left[\begin{array}{ccc}
-3+\xi & -2 & -2 \\
2 & 1+\xi & 2 \\
2 & 2 & 1+\xi
\end{array}\right]
$$

We then define

$$
K_{n+1}(\xi)=K_{3}(\xi)=\frac{\hat{R}_{2}(\xi) K_{2}(\xi)}{\xi-0.86+i 0.5}=\left[\begin{array}{cc}
0.86+\xi & -0.5 \\
0.5 & 0.86+\xi \\
0 & 0 \\
0 & 0
\end{array}\right] \begin{gathered}
\text { Observe that } F \text { is Hurwitz, and moreover that } Z(\xi)= \\
F(-\xi)^{T}\left[\begin{array}{cc}
-3 & 0 \\
0 & 1
\end{array}\right] F(\xi) . \\
\text { V. COMMENTS }
\end{gathered}
$$

As stated in Theorem 3, the matrix $K_{n+1}$ has the last p rows equal to zero.

Observe that $Q_{n+1}(\xi)=\left[\begin{array}{cc}0.86+\xi & -0.5 \\ 0.5 & 0.86+\xi\end{array}\right]$ has singularities in $-0.86 \pm i 0.5$, i.e. $Q_{n+1}$ is Hurwitz. Moreover, as stated in the last part of Theorem $3, Q_{n+1}(-\xi)^{T} Q_{n+1}(\xi)=$ $Z(\xi)$, i.e. $Q_{n+1}$ is a spectral factor of $Z$.

Example 5: The purpose of this example is to show that the ideas presented in this paper can be used also for the general case, provided certain preprocessing steps are carried out. We consider an example of a mixed-sensitivity problem from [15], Example 4.4.3, with parameters $r=0, c=1$, and $\gamma=2$.

Consider the matrix

$$
\begin{aligned}
Z(\xi) & =\left[\begin{array}{cc}
1 & -1+\xi \\
-1 & 0 \\
2 & 2 \xi
\end{array}\right]^{T}\left[\begin{array}{ccc}
1 & 0 & 0 \\
0 & 1 & 0 \\
0 & 0 & -1
\end{array}\right]\left[\begin{array}{cc}
1 & -1-\xi \\
-1 & 0 \\
2 & -2 \xi
\end{array}\right] \\
& =\left[\begin{array}{cc}
-2 & -1+3 \xi \\
-1-3 \xi & 1+3 \xi^{2}
\end{array}\right]
\end{aligned}
$$

It can be shown that $Z(i \omega)$ has inertia $(1,0,1)$ for all $\omega \in \mathbb{R}$. However, it can be readily verified that the transfer function associated with the factorization above is not strictly proper.

In order to perform the spectral factorization, we write

$$
N(\xi)=\left[\begin{array}{ll}
2 & -2 \xi
\end{array}\right]=\left[\begin{array}{ll}
2 & 0
\end{array}\right]\left[\begin{array}{cc}
-1 & -1-\xi \\
-1 & 0
\end{array}\right]+\left[\begin{array}{ll}
0 & 2
\end{array}\right]
$$

We discuss some of the issues pertaining to Algorithm 2 in a series of remarks centered around various aspects of the result of Theorem 3 .

Remark 6: As mentioned in the introduction to the previous section, in the illustration of out results we have decided to concentrate on a special case for ease of exposition. Observe for instance that in Example 5 we have considered a matrix $Z$ which did not satisfy the assumptions stated in Theorem 3, and that with minimal adaptation Algorithm 2 has been shown to work even in that case. In a forthcoming paper we will show that the only necessary and sufficient condition for the existence of a $\Sigma_{1}$-spectral factorization and for the functioning of Algorithm 2 are Assumptions (1) and (2). This provides a necessary and sufficient condition for the existence of a $\Sigma_{1}$-spectral factorization which, to the best of the authors' knowledge, is original and alternative to the ones already known in the literature (see [5]).

Remark 7: It is well-known (see for example Theorem 1 of [19]) that the problem of $J$-spectral (rational) factorization is related to that of solving algebraic Riccati equations with indefinite input cost matrices $R$. In a forthcoming publication we will pursue from the point of view adopted in this paper this relationship and the application of interpolation algorithms to the state-space case.

Remark 8: Algorithm 2 has a clear interpretation from the point of view of QDFs and their calculus. In particular, it can be shown that the model (2) corresponds to a very special, 
"matched" (in the sense of section 10 of [23]) basis of the state space of $\operatorname{Im} K\left(\frac{d}{d t}\right)$ and consequently of $Q_{\Phi}$, and that the iterations of the algorithm correspond to the determination of special QDFs satisfying certain interpolation conditions. These connections are the subject of a forthcoming publication.

Remark 9: The numerical and computational complexity aspects of the implementation of Algorithm 2 are a topic of considerable interest in its own right, as it is its comparison with existing algorithms (see for example [14]); these issues will be addressed elsewhere.

Remark 10: One of the most interesting aspects of Algorithm 2 is the following. It can be shown that the iteration produces, in the last $\mathrm{p}$ rows of the model $\hat{R}_{n+1}$, a kernel representation of an optimal controller for an $H_{\infty}$-problem associated with $\Sigma$ and $K$ of the symmetric canonical factorization of $\Phi(\zeta, \eta)$. This fact opens a new avenue of approach to the solution of the kind of $H_{\infty}$-control problems arising in the polynomial- and in the behavioral approach to control, an approach based on vector-exponential time-series modeling, or equivalently on interpolation. The connection of $\mathrm{H}_{\infty}$ control and interpolation problems has long been recognized (see [9], [10], [11], [12]). The results presented in this paper can be shown to have these well-known techniques developed in the transfer function and in the state-space frameowrk as special cases. The authors will pursue these ramifications in a forthcoming paper, in which also concrete applications of the results presented here to the design of $H_{\infty}$-controllers will be considered.

\section{ACKNOWLEDGMENT}

The third author thanks the Netherlands Organization for Scientific Research (NWO) for financially supporting the visit to IIT Bombay during which the results presented in this paper were obtained.

\section{REFERENCES}

[1] F.A. Aliev and V.B. Larin, "Algorithm of $J$-spectral factorization of polynomial matrices", Automatica, vol. 33, no. 12, pp. 2179-2182, 1997

[2] B.D.O. Anderson, K.L. Hitz, and N.D. Diem, "Recursive algorithm for spectral factorization", IEEE Trans. Circ. Syst., vol. 21, no. 6, pp. 453-464, Nov. 1976.

[3] Antoulas, A.C. and Willems, J.C., "A behavioral approach to linear exact modeling", IEEE Trans. Aut. Contr., vol. 38, pp. 1776-1802, 1993.

[4] A. Barabanov, "Canonical matrix factorization and polynomial Riccati equations", European J. Control, col. 3, no. 1, pp. 47-67, 1997.

[5] W. Coppel, Linear Systems, Notes in Pure Mathematics,vol. 6, Australian National University, Canberra, 1972.

[6] T.T. Georgiou and P.P. Khargonekar, "Spectral factorization and Nevanlinna-Pick interpolation", SIAM J. on Control and Optimization, vol. 25, no. 3, pp. 754-766, 1987.

[7] T.T. Georgiou and P.P. Khargonekar, "Spectral factorization of matrix valued functions using interpolation theory", IEEE Trans. on Circuits and Systems, vol. 36, no. 4, pp. 568-574, 1989.

[8] J. Ježek and V. Kučera, "Effient algorithm for matrix spectral factorization", Automatica, vol. 21, no. 6, pp. 663-669, 1985.

[9] H. Kimura, "Directional interpolation approach to $H_{\infty}$-Optimization and robust stabilization", IEEE Trans. Aut. Contr., vol. 32, no. 12, pp. 1085-1093, 1987.

[10] H. Kimura, "Directional interpolation in the state space", Systems and Control Letters, vol. 10, pp. 317-324, 1988.
[11] H. Kimura, "Conjugation, interpolation, and model-matching in $H_{\infty}$ ", International J. Control, vol. 49, pp. 269-307, 1989.

[12] H. Kimura, "Application of classical interpolation theory", in N. Nagai, Ed., Linear Circuits, Systems, and Signal Processing, pp. 6185, Marcel Dekker Inc., NY, 1990.

[13] H. Kwakernaak, "The polynomial approach to $H_{\infty}$-optimal regulation", in $H_{\infty}$-control theory, E. Mosca and L. Pandolfi, Eds., Lecture Notes in Mathematics 1496, Springer-Verlag, Berlin, 1991.

[14] H. Kwakernaak and M. Šebek, "Polynomial $J$-spectral factorization", IEEE Trans. Aut. Contr., vol. 39, no. 2, pp. 315-328, 1994.

[15] G. Meinsma, Frequency-domain methods in $H_{\infty}$-control, Doctoral Dissertation, University of Twente, 1993.

[16] Polderman, J.W. and Willems, J.C., Introduction to mathematical system theory: a behavioral approach, Springer-Verlag, Berlin, 1997.

[17] A.C.M. Ran, "Necessary and sufficient conditions for existence of $J$ spectral factorization for para-Hermitian rational matrix functions", Automatica, vol. 39, pp. 1935-1939, 2003.

[18] A.C.M. Ran and L. Rodman, "Factorization of matrix polynomials with symmetries", SIAM Journal on Matrix Analysis and Applications, vol. 15, pp. 845-864, 1994.

[19] A.C.M. Ran, "Necessary and sufficient conditions for existence of Jspectral factorization for para-Hermitian rational matrix functions", Automatica, vol. 39, no. 11, pp. 1935-1939, 2003.

[20] Rapisarda, P. and Willems, J.C.,"The subspace Nevanlinna interpolation problem and the most powerful unfalsified model", System and Control Letters, vol. 32, pp. 291-300, 1997.

[21] H.L. Trentelman and P. Rapisarda, "New algorithms for polynomial $J$ spectral factorization", Mathematics of Control, Signals and Systems, vol. 12, pp. 24-61, 1999.

[22] Willems, J.C., "From time series to linear system, part II: Exact modeling", Automatica, vol. 22, pp. 675-694, 1986.

[23] Willems, J.C. and Trentelman, H.L., "On quadratic differential forms", SIAM J. Control Opt., vol. 36, no. 5, pp. 1703-1749, 1998.

[24] J.C. Willems and H.L. Trentelman, " $H_{\infty}$-control in a behavioral context: The full information case", IEEE Trans. Aut. Contr., vol. 44, pp. 521-536, 1999.

[25] J.C. Willems and H.L. Trentelman, "Synthesis of dissipative systems using quadratic differential forms, Part I", IEEE Trans. Aut. Contr., vol. 47, pp. 53-69, 2002. 